$09 ; 11$

\title{
Анализ фокусировки волн в усеченной линзе Гутмана
}

\author{
() Е.И. Семерня ${ }^{1}$, С.П. Скобелев ${ }^{1,2}$ \\ ${ }^{1}$ Московский фризико-технический институт (Национальный исследовательский университет), \\ Долгопрудный, Московская обл., Россия \\ ${ }^{2}$ ПАО „Радиосизика“, Москва, Россия \\ E-mail: poshisholina@gmail.com
}

Поступило в Редакцию 14 января 2021 г.

В окончательной редакции 5 мая 2021 r.

Принято к публикации 7 мая 2021 г.

Рассмотрена задача рассеяния плоской электромагнитной волны на усеченной осесимметричной линзе Гутмана, включающая расчет поля внутри линзы. Задача решена численно с использованием гибридного проекционного метода, разработанного ранее для общего случая рассеяния волн на неоднородных телах вращения. Приведены новые результаты, демонстрирующие точность метода, а также распределения поля в линзе Гутмана, характеризующие особенности фокусировки волны в зависимости от размера линзы, фокусного расстояния и положения плоскости усечения.

Ключевые слова: неоднородные среды, тела вращения, линза Гутмана, фокусировка волн, численные методы.

DOI: 10.21883/PJTF.2021.15.51235.18709

Линза Гутмана [1], представляющая собой частное решение общей задачи Люнеберга, фокусирует поле падающей плоской волны в точку, расположенную внутри линзы, а не на ее поверхности, как это имеет место в обычной линзе Люнеберга. Согласно [1], профиль ее относительной диэлектрической проницаемости в направлении радиальной координаты $r$ можно записать в виде

$$
\varepsilon(r)=1+\frac{a^{2}-r^{2}}{f^{2}},
$$

где $a$ - радиус линзы, $f \leqslant a-$ фокусное расстояние. Применение линзы Гутмана в антенной технике позволяет уменьшить размер антенны по сравнению с таковым для линзы Люнеберга. Однако если требуется обеспечить сканирование луча путем механического перемещения облучателя по сферической поверхности внутри линзы, то конструкция антенны сильно усложняется. Существенное упрощение конструкции достигается путем отсечения части линзы плоскостью, касательной к фокальной сфере. Этот подход позволяет дополнительно уменьшить продольный размер антенны, а сканирование лучом теперь может осуществляться перемещением облучателя вдоль плоской части поверхности линзы, образовавшейся в результате усечения.

Численное моделирование цилиндрической многослойной усеченной линзы Гутмана в двумерном случае рассмотрено в [2], а исследование осесимметричной усеченной линзы, изготовленной из искусственного диэлектрика, проведено в [3]. Результаты, полученные в [2,3], представляют большой интерес, однако влияние усечения линзы на фокусировку плоской волны там рассмотрено не было. В настоящей работе такое рассмотрение проводится с использованием решения задачи рассеяния плоской волны на осесимметричной линзе
Гутмана - как усеченной, так и исходной. Численное решение получено с использованием гибридного проекционного метода, разработанного в [4] для общего случая рассеяния волн на неоднородных телах вращения.

Продольное сечение усеченной осесимметричной линзы, расположенной в свободном пространстве в декартовых координатах $x, y, z$ и сферических координатах $r, \theta, \varphi$, показано на вставках к рис. 1 и 2. Линза характеризуется радиусом $a$, а плоскость усечения, перпендикулярная оси $z$, расположена на расстоянии $a_{1}$ от центра сферы, совпадающего с началом координат. Относительная магнитная проницаемость среды везде считается равной единице.

Предположим, что источники возбуждения расположены на некотором расстоянии от линзы. Тогда, взяв гармоническую зависимость полей от времени в виде $e^{-i \omega t}$, можно представить поперечное электрическое и магнитное поле во внешней области, где $r \geqslant a$, как разложение по сферическим волновым функциям

$$
\begin{aligned}
\mathbf{E}_{\tau}(r, \theta, \varphi)= & \frac{1}{k r} \sum_{q=0}^{\infty} \sum_{m=-q}^{q}\left\{\left[A_{1 q}^{m} \psi_{q}(k r)+R_{1 q}^{m} \zeta_{q}(k r)\right] \mathbf{Y}_{1 q}^{m}\right. \\
& \left.+\left[A_{2 q}^{m} \psi_{q}^{\prime}(k r)+R_{2 q}^{m} \zeta_{q}^{\prime}(k r)\right] \mathbf{Y}_{2 q}^{m}\right\}, \\
\mathbf{H}_{\tau}(r, \theta, \varphi)= & -\frac{i}{\eta_{0} k r} \sum_{q=0}^{\infty} \sum_{m=-q}^{q}\left\{\left[A_{1 q}^{m} \psi_{q}^{\prime}(k r)+R_{1 q}^{m} \zeta_{q}^{\prime}(k r)\right] \mathbf{Y}_{2 q}^{m}\right. \\
& \left.+\left[A_{2 q}^{m} \psi_{q}(k r)+R_{2 q}^{m} \zeta_{q}(k r)\right] \mathbf{Y}_{1 q}^{m}\right\},
\end{aligned}
$$

где $A_{1 q}^{m}$ и $A_{2 q}^{m}$ - амплитуды ТЕ- и ТМ-волн, излученных источниками, $R_{1 q}^{m}$ и $R_{2 q}^{m}-$ неизвестные амплитуды ТЕ- и ТМ-волн, рассеянных телом, $\psi_{q}(k r)$ - функции Риккати-Бесселя, $\zeta_{q}(k r)-$ функции Риккати-Ханкеля 
первого рода, $\mathbf{Y}_{j q}^{m}(\theta, \varphi)$ - ортонормированные поперечные векторные сферические функции [4], определяемые присоединенными функциями Лежандра $(j=1,2)$, $k=2 \pi / \lambda-$ волновое число, $\lambda-$ длина волны в свободном пространстве, $\eta_{0}-$ сопротивление свободного пространства.

Поперечное электрическое поле и поперечное магнитное поле внутри сферической области $0 \leqslant r \leqslant a$, содержащей линзу, также представляются в виде разложений по поперечным векторным функциям

$$
\begin{gathered}
\mathbf{E}_{\tau}(r, \theta, \varphi)=\sum_{q=0}^{\infty} \sum_{m=-q}^{q}\left[E_{1 q}^{m}(r) \mathbf{Y}_{1 q}^{m}+E_{2 q}^{m}(r) \mathbf{Y}_{2 q}^{m}\right], \\
\mathbf{H}_{\tau}(r, \theta, \varphi)=\frac{1}{\eta_{0}} \sum_{q=0}^{\infty} \sum_{m=-q}^{q}\left[H_{1 q}^{m}(r) \mathbf{Y}_{2 q}^{m}+H_{2 q}^{m}(r) \mathbf{Y}_{1 q}^{m}\right]
\end{gathered}
$$

с неизвестными переменными коэффициентами, зависящими от продольной координаты.

Проектируя уравнения Максвелла, которым должны удовлетворять поля (4) и (5), на поперечные функции так, как описано в [4], получим систему обыкновенных дифференциальных уравнений для функций $U_{j q}(r)=k r E_{j q}^{m}(r)$ и $V_{j q}(r)=k r H_{j q}^{m}(r), j=1,2$, для каждого $m$ с матричными элементами, описанными в [4]. Дифференциальные уравнения далее решаются одномерным методом конечных элементов, как описано в [4]. При этом неизвестные переменные коэффициенты представляются в виде разложений

$$
U_{j q}(r)=\sum_{n=1}^{N} U_{j n q} f_{n}(r), \quad V_{2 q}(r)=\sum_{n=1}^{N} V_{2 n q} f_{n}(r),
$$

где $U_{j n q}$ и $V_{2 n q}-$ неизвестные постоянные коэффициенты, $N$ - число узлов с координатами $r_{n}=n \Delta, \Delta=a / N$, a $f_{n}(r)$ - треугольные функции с вершинами, расположенными в указанных узлах. Проектируя указанные дифференциальные уравнения на треугольные функции $f_{n^{\prime}}(r)[4]$, сводим их к системе линейных алгебраических уравнений

$$
\begin{gathered}
\sum_{n=1}^{N} \sum_{q=|m|}^{\infty}\left(\widetilde{W}_{1 n^{\prime} n}^{p q} U_{1 n q}-\widetilde{X}_{n^{\prime} n}^{p q} U_{2 n q}\right)+\zeta_{p}^{\prime}(k a) R_{1 p}^{m} \delta_{n^{\prime} N} \\
=-A_{1 p}^{m} \psi_{p}^{\prime}(k a) \delta_{n^{\prime} N}, \\
\sum_{n=1}^{N} \sum_{q=|m|}^{\infty}\left(\widetilde{X}_{n^{\prime} n}^{p q} U_{1 n q}+\widetilde{W}_{2 n^{\prime} n}^{p q} U_{2 n q}\right)+i \sum_{n=1}^{N} K_{n^{\prime} n}^{(1)} V_{2 n p} \\
-\xi_{p}(k a) R_{2 p}^{m} \delta_{n^{\prime} N}=A_{2 p}^{m} \psi_{p}(k a) \delta_{n^{\prime} N}, \\
-i \sum_{n=1}^{N} K_{n^{\prime} n}^{(2)} U_{2 n p}+\sum_{n=1}^{N} \sum_{q=|m|} \widetilde{Z}_{n^{\prime} n}^{p q} V_{2 n q}=0,
\end{gathered}
$$

где $\delta_{n^{\prime} N}$ - символ Кронекера, выражения для матричных коэффициентов $\widetilde{W}_{1 n^{\prime} n}^{p q}, \widetilde{X}_{n^{\prime} n}^{p q}, \widetilde{Z}_{n^{\prime} n}^{p q}$ приведены в [4].
Алгебраические уравнения (7)-(9) получены с учетом непрерывности магнитных полей (3) и (5) при $r=a$. Условие непрерывности электрических полей (2) и (4) при $r=a$ дает еще два соотношения для системы уравнений

$$
\begin{aligned}
& U_{1 N p}-\xi_{p}(k a) R_{1 p}^{m}=A_{1 p}^{m} \psi_{p}(k a), \\
& U_{2 N p}-\xi_{p}^{\prime}(k a) R_{2 p}^{m}=A_{2 p}^{m} \psi_{p}^{\prime}(k a) .
\end{aligned}
$$

Размерность системы уравнений (7)-(11) после усечения равна $(3 N+2) L$, где $L-$ количество учитываемых меридиональных гармоник в (2), (3), (4) и (5) для каждого $m$. После решения усеченной системы можно вычислить поле в каждой точке пространства, включая распределение поля внутри усеченной линзы Гутмана, которое представляет наибольший интерес.

Алгоритм, описанный выше, был реализован в среде MATLAB. Работа программы была проверена путем сравнения ее результатов с результатами, полученными методом интегральных уравнений, реализованным в CST Microwave Studio, для случая однородной усеченной сферы, возбуждаемой плоской волной круговой поляризации, распространяющейся вдоль отрицательного направления оси $z$. Результаты сравнения для усеченной сферы с параметрами $a=2.5 \lambda, a_{1}=0.625 a$ и $\varepsilon=3.56$ приведены на рис. $1, a$, где показано распределение поля вдоль оси $z$, и на рис. $1, b$, где показана бистатическая эффективная площадь рассеяния; НРМ соответствует сокращению от Hybrid Projection Method (гибридный проекционный метод). Результаты в CST были получены с использованием около 33000 треугольных сегментов, сформированных на поверхности сферы. Начальные значения для $N$ и $L$ в НРМ определяются формулами $N=[50 a / \lambda]$ и $L=5+[1.5 k a]$, где [...] означает целую часть числа, с их последующим увеличением для достижения стабилизации результатов. Результаты, показанные на рис. 1 , соответствуют $N=200$ и $L=41$.

Результаты применения гибридного проекционного метода к расчету распределения поля на оси $z$ и в поперечных плоскостях, проходящих через максимумы поля, в усеченной линзе Гутмана с $a=2.5 \lambda$ для значений фокусного расстояния $f=0.8 a$ и $0.625 a$, полученные при тех же $N$ и $L$, что и в предыдущем случае, приведены на рис. $2, a$ и $b$. Аналогичные результаты для $a=1.5 \lambda$, полученные при $N=150$ и $L=31$, показаны на рис. 2, $c$ и $d$. Полученные результаты сравниваются с аналогичными результатами, соответствующими обычной линзе Гутмана, для которой $a_{1}=a$. Сравнение показывает, что усечение линзы плоскостью, проходящей через фокус, приводит к образованию осцилляций поля внутри линзы, вызванных наличием отражений волн от плоской части поверхности, рассогласованной со свободным пространством. Поскольку, согласно (1), уменьшение фокусного расстояния соответствует увеличению значения проницаемости в точке фокуса, рассогласование увеличивается и уровень осцилляций повышается. Еще один эффект состоит в некотором 

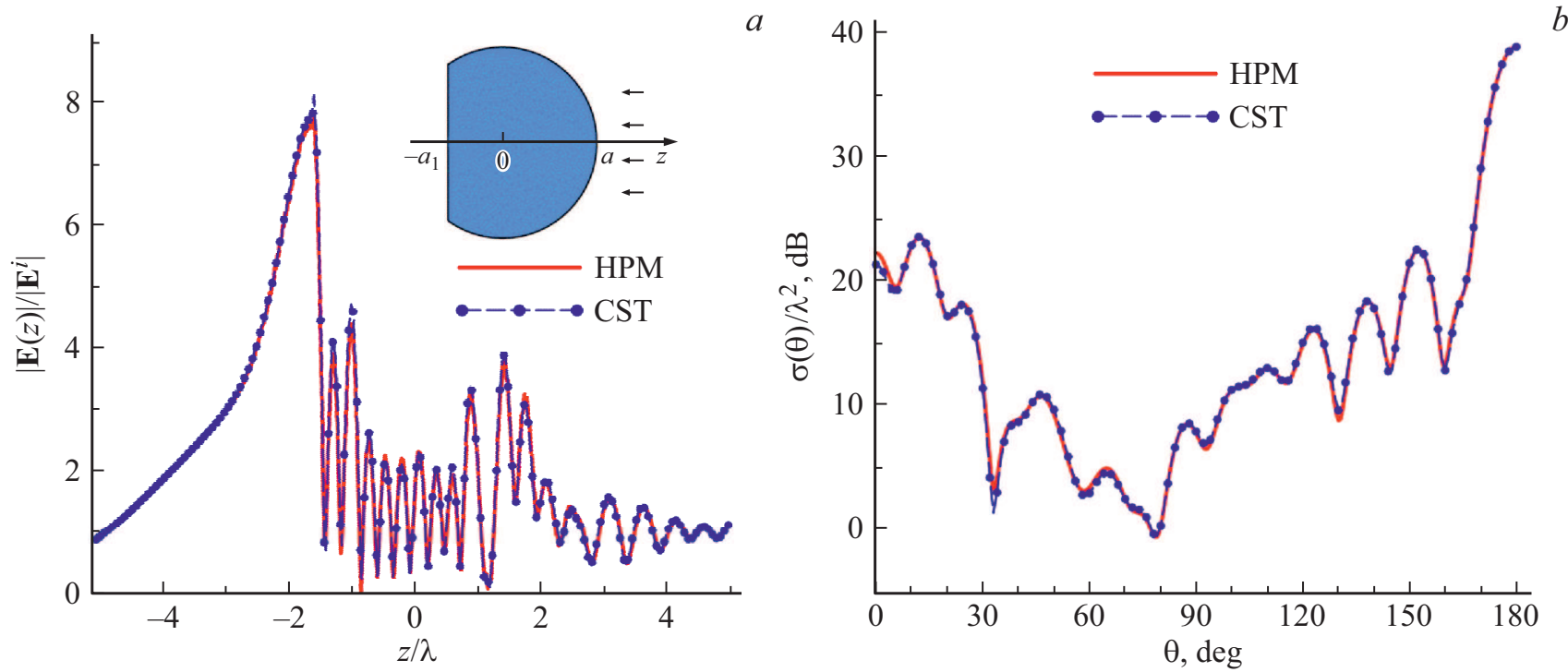

Рис. 1. Нормированное распределение поля вдоль оси $z$ однородной усеченной сферы $(a)$ и бистатическая эффективная площадь рассеяния последней $(b)$.
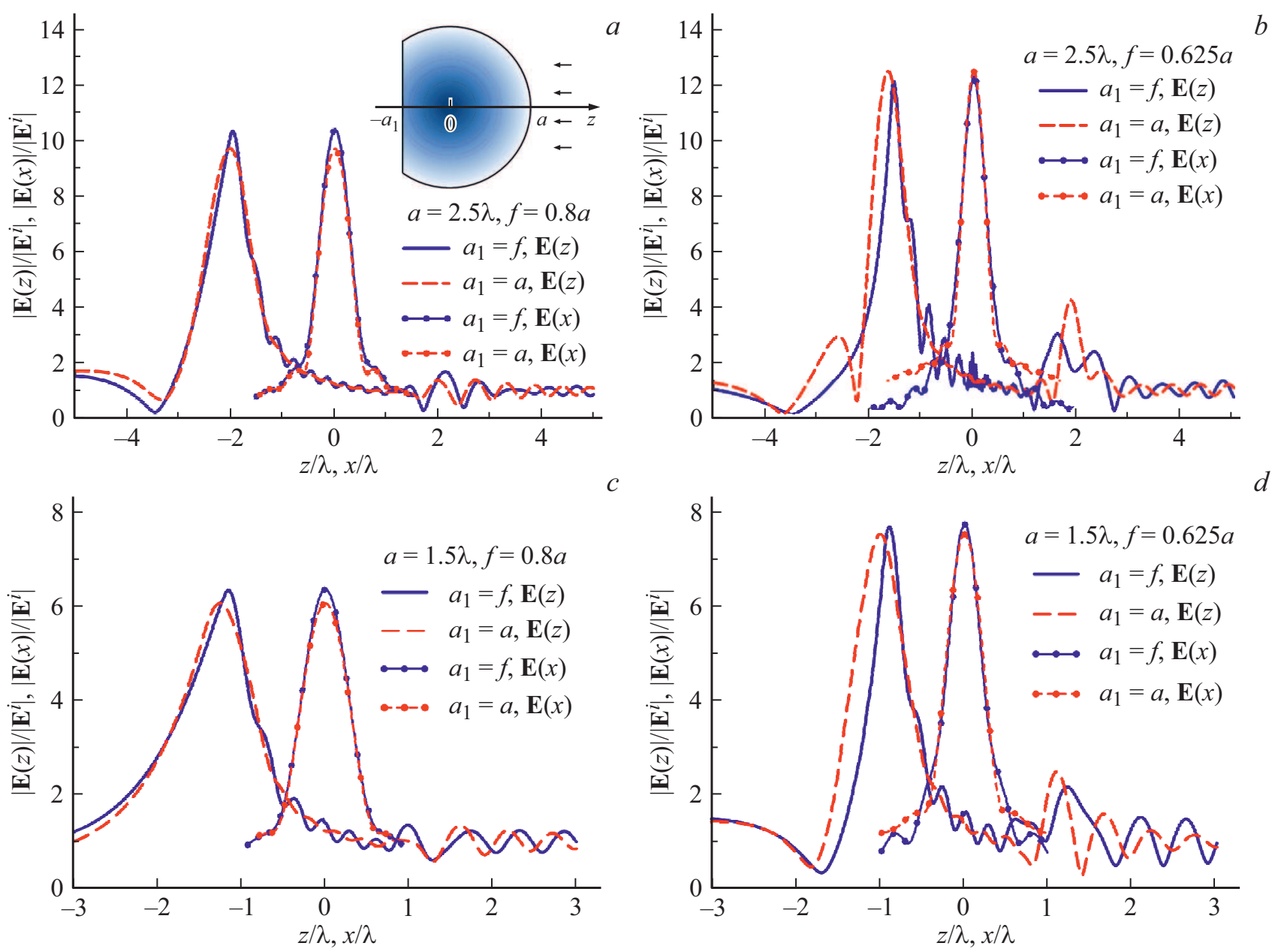

Рис. 2. Нормированные распределения поля вдоль оси $z$ и в поперечных плоскостях, проходящих через максимумы поля, в линзе Гутмана с $a=2.5 \lambda(a, b)$ и $1.5 \lambda(c, d)$. 
смещении максимума поля в сторону центра линзы по сравнению со случаем обычной линзы Гутмана. Выявленные особенности фокусировки могут оказаться полезными при проектировании облучателя линзы.

\section{Конфликт интересов}

Авторы заявляют, что у них нет конфликта интересов.

\section{Список литературы}

[1] A.S. Gutman, J. Appl. Phys., 25 (7), 855 (1954). DOI: $10.1063 / 1.1721757$

[2] O. Quevedo-Teruel, W. Tang, Y. Hao, Opt. Lett., 37 (23), 4850 (2012). DOI: 10.1364/OL.37.004850

[3] O. Bjorkqvist, O. Zetterstrom, O. Quevedo-Teruel, Electron. Lett., 55 (25), 1318 (2019). DOI: 10.1049/el.2019.2483

[4] E.I. Semernya, S.P. Skobelev, J. Opt. Soc. Am. A., 37 (12), 1873 (2020). DOI: 10.1364/JOSAA.396534 